\title{
Novel surfactant-assistant hydrothermal fabrication of lignin microsphere as a green reducer and carrier for Pd nanoparticles
}

\author{
Tairan Pang a , Guanhua Wang a ${ }^{*}$, Jing Ge ${ }^{a}$, Ni Wei a , Wenjie Sui ${ }^{b^{*}}$, Ashak Mahmud Parvez ${ }^{c, d}$, \\ Chuanling $\mathrm{Si}^{\text {a }}$ \\ a Tianjin Key Laboratory of Pulp and Paper, College of Light Industry Science and Engineering, \\ Tianjin University of Science and Technology, Tianjin 300457, China \\ ${ }^{\mathrm{b}}$ State Key Laboratory of Food Nutrition and Safety, College of Food Science and Engineering, \\ Tianjin University of Science and Technology, Tianjin 300457, China \\ ${ }^{c}$ Institute of Combustion and Power Plant Technology (IFK), University of Stuttgart, \\ Pfaffenwaldring 23, D-70569 Stuttgart, Germany \\ ${ }^{\mathrm{d}}$ LRGP, CNRS, ENSIC, 1 rue Gradville 54000 Nancy, France \\ * Corresponding author. Tel.: +86 02260601313 \\ Address: No.29 at 13th Avenue, TEDA, Tianjin 300457, China \\ E-mail address: ghwang@tust.edu.cn (Guanhua Wang) \\ wjsui@tust.edu.cn (Wenjie Sui)
}

Contents

Thirteen pages (including cover sheet)

Eight figures (Figure S1, S2, S3, S4, S5, S6, S7 and S8)

Two tables (Table S1 and S2) 


\section{ISOLATION OF KRAFT LIGNIN}

Chips of Populus L. were provided by Huatai Paper Co. Ltd. (Dongying, Shandong Province, China). Poplar powders were obtained by mechanical grinding and screening through a 20 mesh sieve $(0.83 \mathrm{~mm}) .50 \mathrm{~g}$ of poplar powder were added to $400 \mathrm{~mL}$ of distilled water containing $12.6 \mathrm{~g} \mathrm{NaOH}$ and $12.3 \mathrm{~g} \mathrm{Na} 2 \mathrm{~S}$. The cooking process was conducted at $165^{\circ} \mathrm{C}$ for $1 \mathrm{~h}$. After cooking, the reactor was cooled down and the black liquor was obtained using 200 mesh gauze filtration followed by centrifugation at $6000 \mathrm{rpm}$ for $10 \mathrm{~min}$. Afterward, $\mathrm{HCl}$ was added into the black liquor to adjust the $\mathrm{pH}$ to 2 and the resulting precipitate was washed with deionized water to a $\mathrm{pH}$ of 7 to obtain kraft lignin. The compositions of the obtained kraft lignin were $89.68 \%$ of Klason lignin, $3.93 \%$ of acid-soluble lignin, $1.32 \%$ of xylose, $0.65 \%$ of glucose and $2.34 \%$ of ash. Weight-average molecular weight of the kraft lignin was $4630 \mathrm{Da}$ and the polydispersity was 1.60 . Through SEM determination, the kraft lignin exhibited a broad particle size distribution ranging from 90 to $8000 \mathrm{~nm}$.

\section{SEM CHARACTERIZATION}

For the SEM measurement, a small amount of freeze-dried lignin particles was evenly spread on the conductive adhesive and coated with gold before SEM observation (JSM-IT300LV, JEOL, Japan) using a Denton Vacuum Desk V sputtering system in an argon atmosphere. SEM determination with 10,000x amplification at $10 \mathrm{kV}$ acceleration voltage was conducted.

\section{TEM ANALYSIS}

Thank you for your suggestion. For the TEM measurement, the lignin particles 
were dispersed in deionized water via sonication and then dried on carbon-coated copper grids (T11032, Beijing XXBR Technology Co., Ltd) at room temperate. The resulting sample was then imaged using a TEM apparatus (JEM-2100F, JEOL, Japan).

\section{XRD MEASUREMENTS}

X-ray diffraction (XRD) patterns were recorded using a X-ray diffractometer (Rigaku RU-200B) with $\mathrm{Cu} \mathrm{Ka}$ radiation $(40 \mathrm{kV}, 30 \mathrm{~mA})$ at a scanning speed of $5^{\circ} / \mathrm{min}$ in the $2 \theta$ range of $5-85^{\circ}$.

\section{ICP-MS}

Inductively coupled plasma atomic emission spectrometry (ICP, ICAPQ, ThermoFisher Scientific, Germany) was used to measure the palladium content of composite catalysts after nitric acid digestion. For the nitric acid digestion, $30 \mathrm{mg}$ of the composite catalyst was added to concentrated nitric acid $(6 \mathrm{~mL})$ and stirred for 20 minutes. Afterward, the mixture was placed in a closed PTFE vessel and treated for 90 minutes under microwave conditions to make the sample totally dissolved. Finally, the obtained liquid was then diluted by 100 times for ICP analysis. 

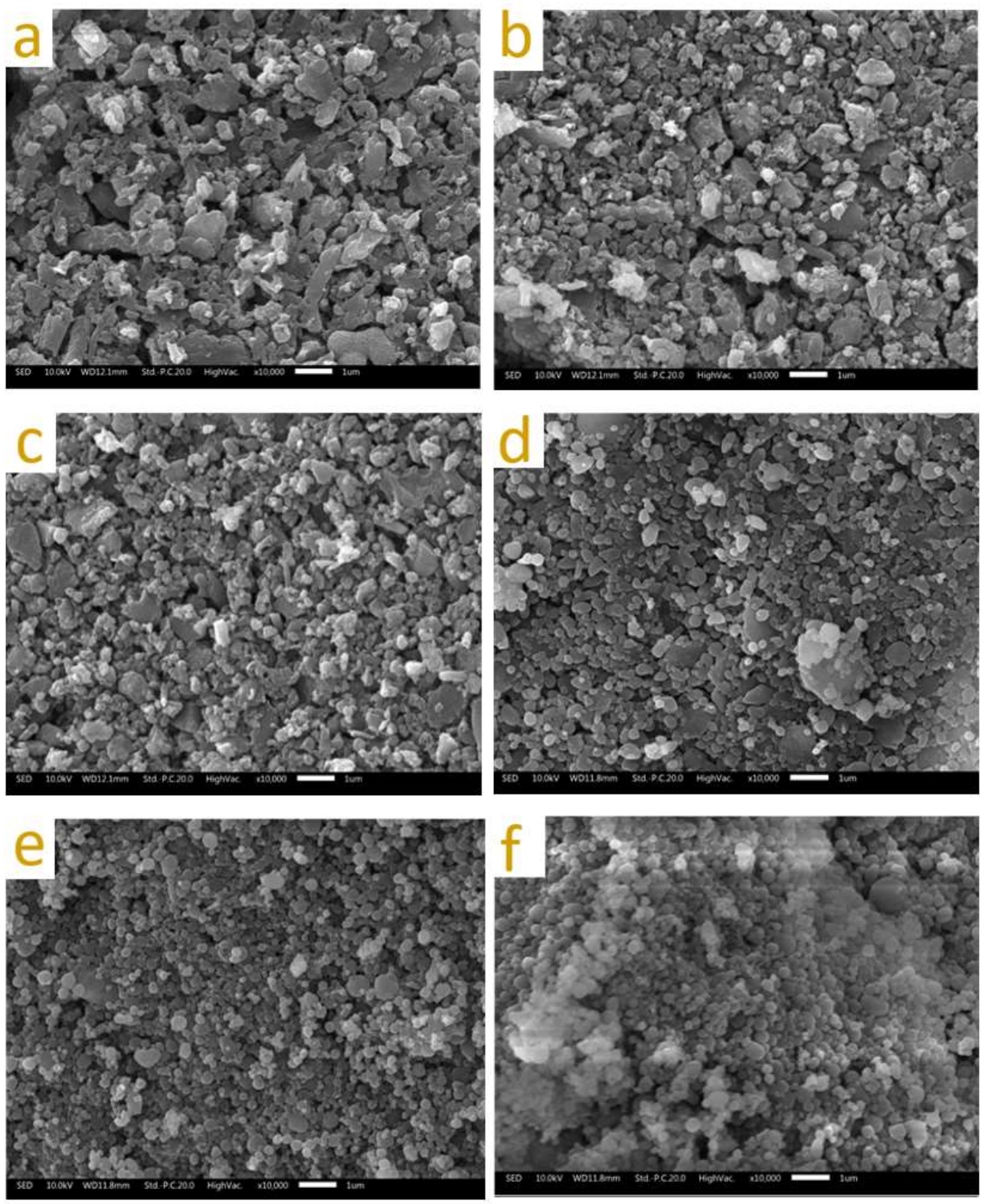

Figure S1. Typical SEM images and size distribution of lignin particles obtained by hydrothermal

treatment at different temperatures: (a) Untreated; Hydrothermal treatment at (b) $120^{\circ} \mathrm{C}$; (c) $140^{\circ} \mathrm{C}$;

(d) $160^{\circ} \mathrm{C}$; (e) $180^{\circ} \mathrm{C}$; and (f) $200^{\circ} \mathrm{C}$. 


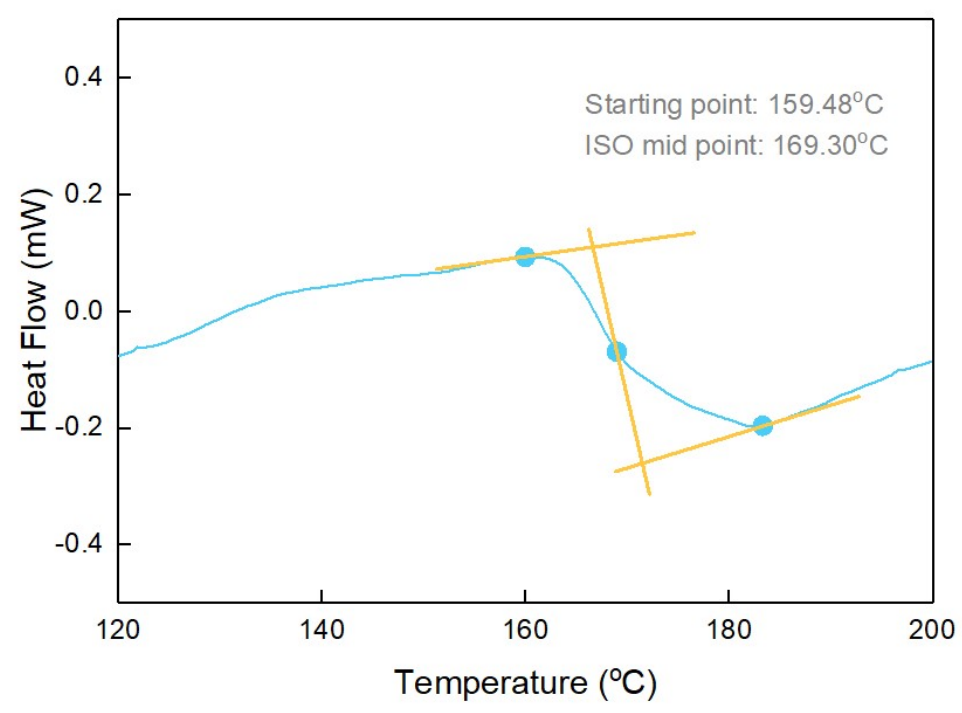

Figure S2. DSC curve of Kraft lignin 

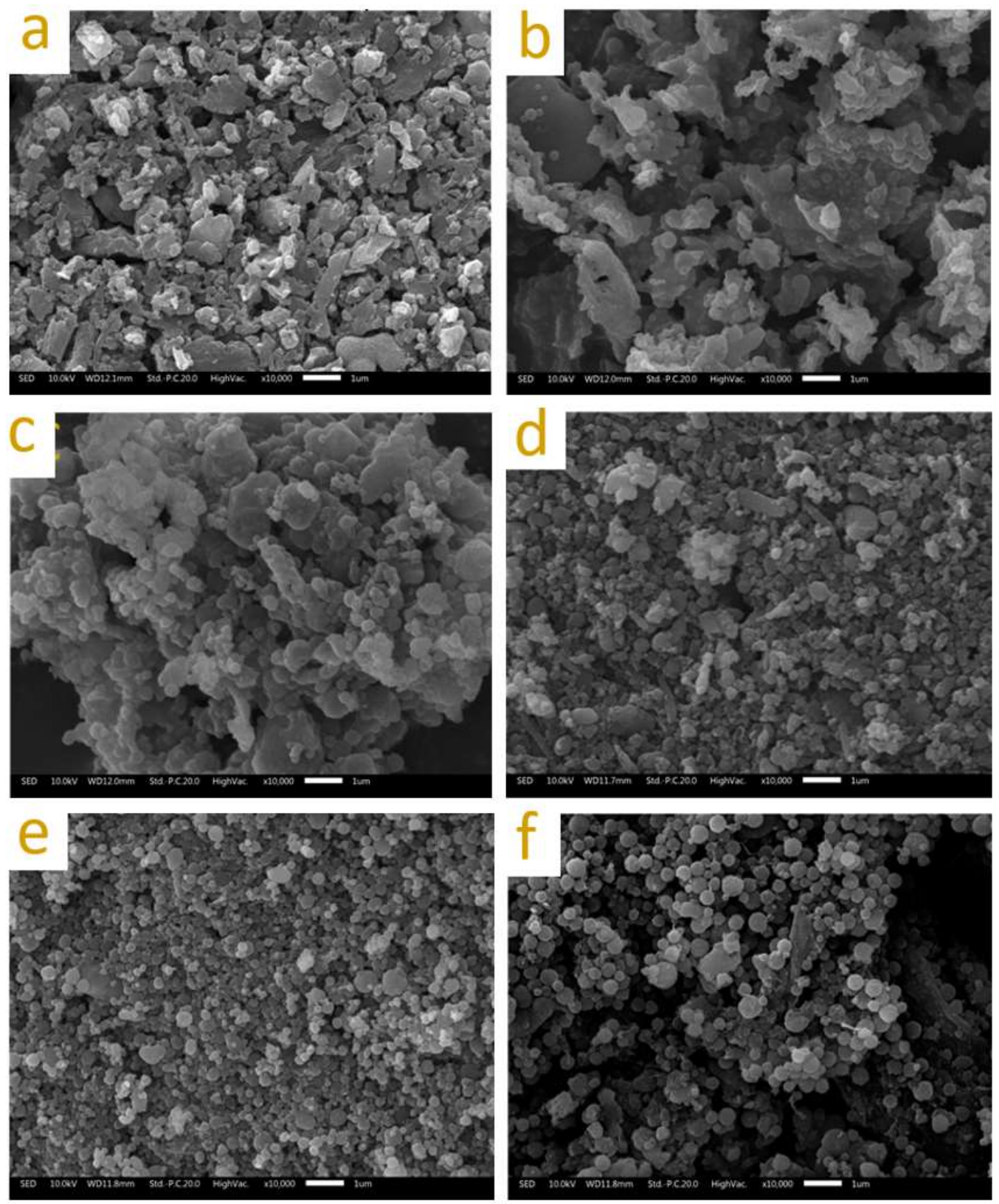

Figure S3. Typical SEM images and size distribution of lignin particles obtained by hydrothermal treatment: a) Untreated; (b) $5 \mathrm{~min}$; (c) $10 \mathrm{~min}$; (d) $15 \mathrm{~min}$; (e) $20 \mathrm{~min}$; and (f) $30 \mathrm{~min}$. 

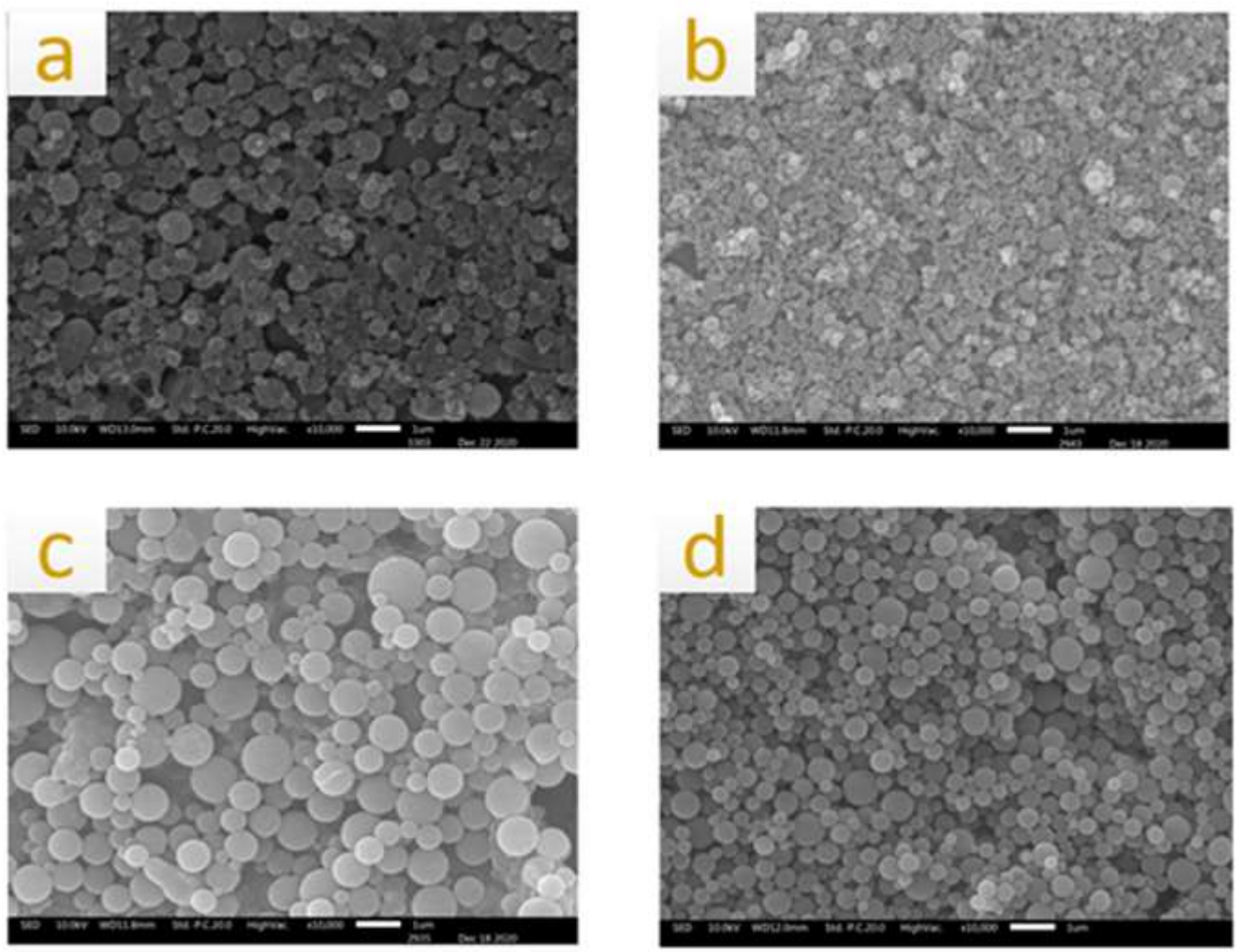

Figure S4. SEM images of LMS obtained by hydrothermal method with addition of different

surfactants in different weight ratio (lignin: surfactant): (a) SLS (1:1); (b) PQA (1:1); (c) PVP (1:1); (d) PVP (1:2). 

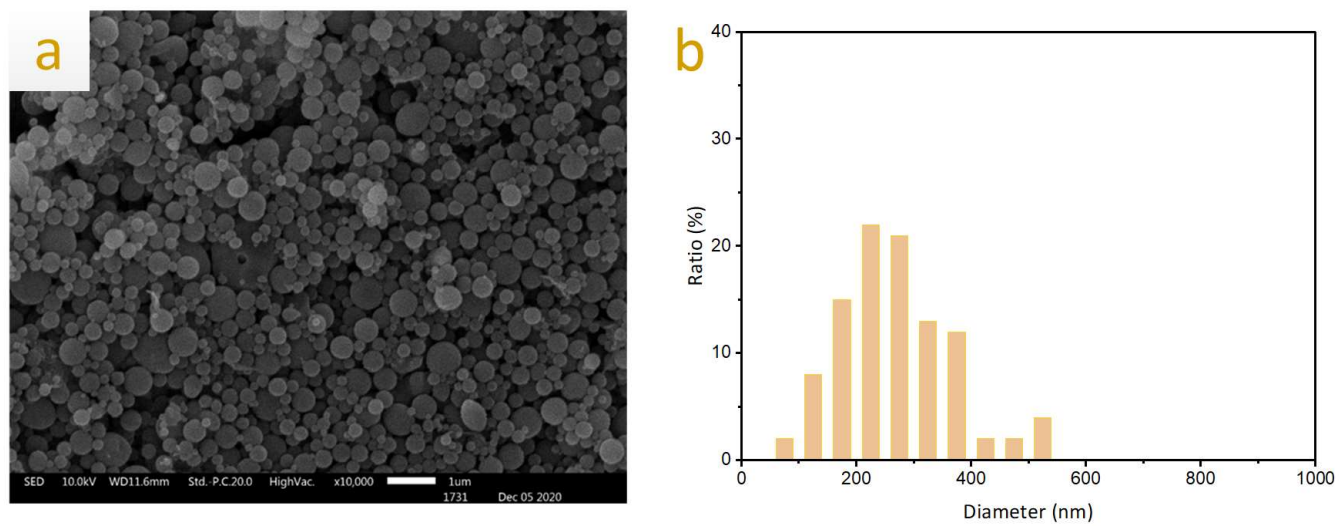

Figure S5. SEM image (a) and size distribution (b) of LMS obtained by hydrothermal method with addition of PVP in 5:1 ratio (PVP to lignin, w/w). 

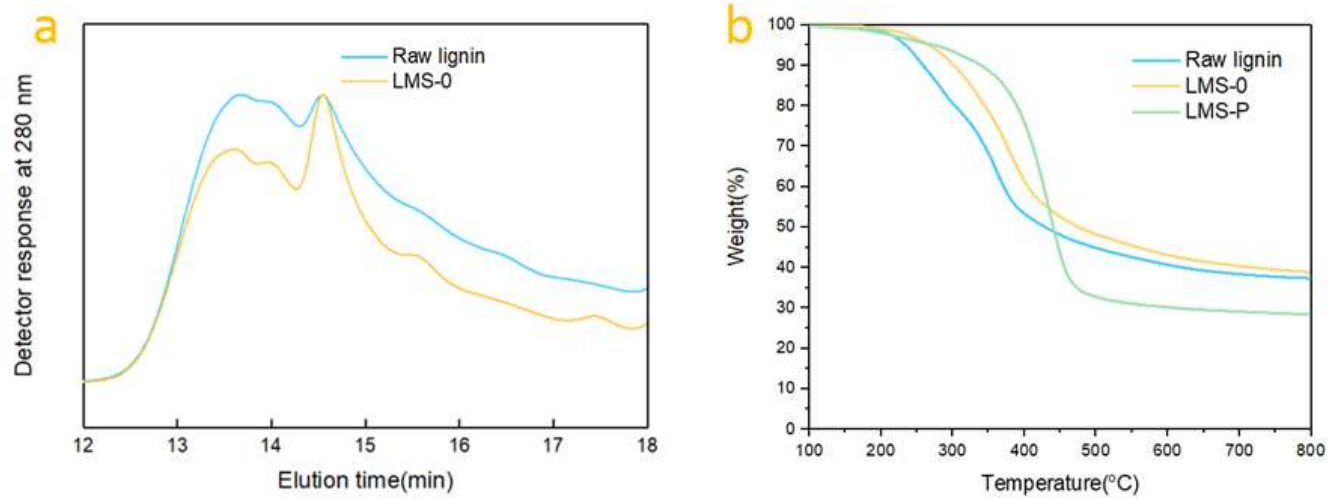

Figure S6. Molecular weight distribution (a) and TG analyses (b) of samples. 
Pd@LMS-0
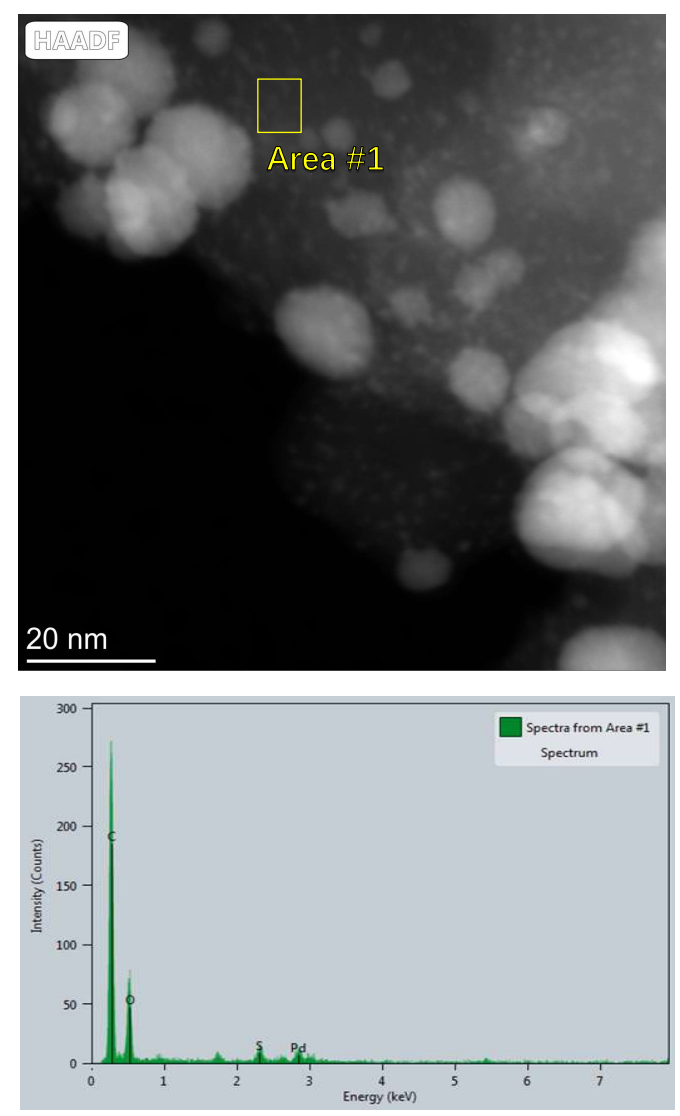

Pd@LMS-P

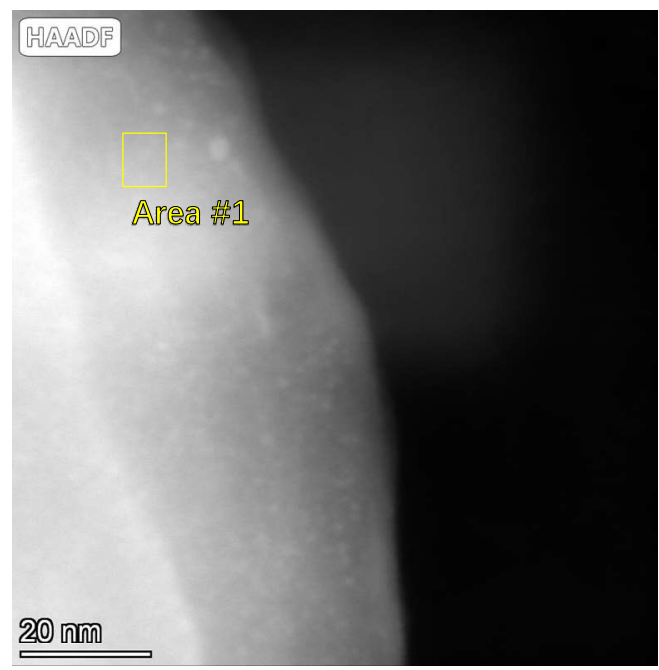

$\square$ Spectra from Area 11

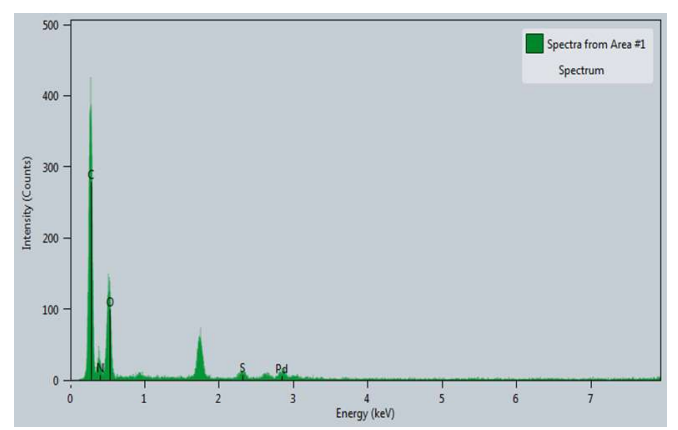

Figure S7. EDX of Pd@LMS-0 and Pd@LMS-P. 


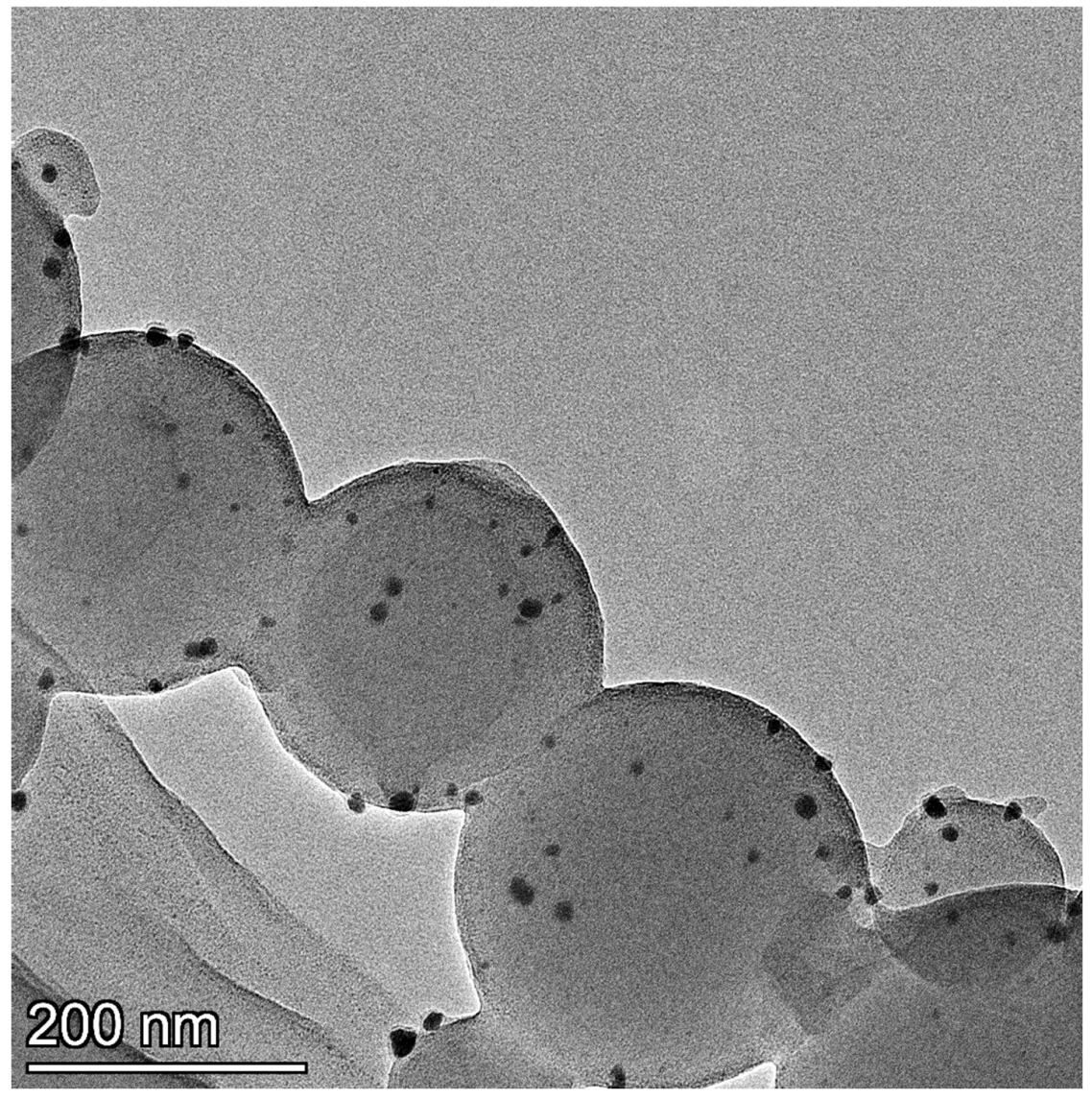

Figure S8. TEM image of Pd@LMS-P after five consecutive runs. 
Table S1. Molecular weight of Kraft lignin and hydrothermal lignin.

\begin{tabular}{cccc}
\hline Lignin samples & $\mathrm{M}_{\mathrm{w}}(\mathrm{g} / \mathrm{mol})$ & $\mathrm{M}_{\mathrm{n}}(\mathrm{g} / \mathrm{mol})$ & PDI \\
\hline Raw lignin & 4630 & 2892 & 1.60 \\
LMS & 4819 & 3171 & 1.52 \\
\hline
\end{tabular}


Table S2. ICP results of Pd@LMS-0 and Pd@LMS-P.

\begin{tabular}{ccccc}
\hline Samples & Weight $(\mathrm{mg})$ & Water $(\mathrm{mL})$ & Pd (KED) (ppb) & Pd content (wt \%) \\
\hline Pd@LMS-0 & 10.1 & 5000 & 377.213 & 18.67 \\
Pd@LMS-P & 9.7 & 5000 & 339.578 & 17.50 \\
\hline
\end{tabular}

